\title{
Shape optimization as a tool to design biocatalytic microreactors
}

Pereira Rosinha Grundtvig, Ines; Daugaard, Anders Egede; Woodley, John; Gernaey, Krist; Krühne, Ulrich

Published in:

Chemical Engineering Journal

Link to article, DOI:

10.1016/j.cej.2017.03.045

Publication date:

2017

Document Version

Peer reviewed version

Link back to DTU Orbit

Citation (APA):

Pereira Rosinha Grundtvig, I., Daugaard, A. E., Woodley, J., Gernaey, K., \& Krühne, U. (2017). Shape optimization as a tool to design biocatalytic microreactors. Chemical Engineering Journal, 322, 215-223. https://doi.org/10.1016/j.cej.2017.03.045

\section{General rights}

Copyright and moral rights for the publications made accessible in the public portal are retained by the authors and/or other copyright owners and it is a condition of accessing publications that users recognise and abide by the legal requirements associated with these rights.

- Users may download and print one copy of any publication from the public portal for the purpose of private study or research.

- You may not further distribute the material or use it for any profit-making activity or commercial gain

- You may freely distribute the URL identifying the publication in the public portal

If you believe that this document breaches copyright please contact us providing details, and we will remove access to the work immediately and investigate your claim. 


\section{Shape optimization as a tool to design biocatalytic microreactors}

Inês P. Rosinha Grundtvig ${ }^{1}$, Anders E. Daugaard ${ }^{2}$, John M. Woodley ${ }^{1}$, Krist V.

Gernaey $^{1}$, Ulrich Krühne ${ }^{1}$

${ }^{1}$ CAPEC-PROCESS Research Center, Department of Chemical and Biochemical Engineering, Technical University of Denmark, Søltofts Plads, Building 229, 2800 Kgs. Lyngby, Denmark

${ }^{2}$ Danish Polymer Centre, Department of Chemical and Biochemical Engineering, Technical University of Denmark, Søltofts Plads, Building 229, 2800 Kgs. Lyngby, Denmark

Corresponding author:

Inês Rosinha Grundtvig

inros@kt.dtu.dk 


\begin{abstract}
Reactor design is commonly constrained to already well-known reactor shapes. This article presents an innovative application of shape optimization techniques to design biocatalytic microreactors. Currently, the optimization of reactor performance is often done by considering solely the process conditions. However, common reactor types used in (bio)chemical processes do not always give the optimal conditions for executing the reaction, and it is therefore necessary to look into new approaches to further improve the performance of reactors. The new application of shape optimization described in this paper has as its main goal the design of a reactor by compensating for the limitations of the reaction system by modifying the reactor configuration. Random search was the optimization method chosen for transforming the initial reactor configuration to a more optimal one.

The case study presented here investigates the impact of a change to the microreactor shape on the active mixing of two parallel streams (one containing an enzyme, amino transaminase, and the other the substrates, acetophenone and isopropylamine) and consequently its influence on the reaction yield. Compared to the original reactor configuration, the shape optimization resulted in changes of the microreactor wall surfaces leading to an 8.4 fold improvement of the reactor yield. This innovative optimization also offers the opportunity to obtain new structures which can later be tested experimentally.
\end{abstract}

Keywords: shape optimization, reactor design, enzymatic reaction, microreactor

\title{
1. Introduction
}

In the chemical industry, bioprocesses have been one of the main areas of industrial expansion in the past decades. Bioprocesses are most commonly applied in the pharmaceutical, the food and the 
chemical industry. Bioprocesses involve the use of microorganisms or their constituent parts such as enzymes - as a catalyst for producing valuable substances such as recombinant proteins, drugs (e.g. insulin, antibiotics) or even bio-based chemicals (e.g. bioethanol) to name a few [1].

The reactor design for a bioprocess is chosen according to available configurations that have been reported in the scientific literature [2], or that are available at the production sites (e.g. stirred tank reactors, plug-flow reactors, packed bed reactors and fluidized bed reactors). After the reactor configuration has been chosen, the process conditions are optimized. This optimization is typically done by adjusting process parameters such as concentration of reactants, temperature, feed flowrate and $\mathrm{pH}$ value.

Nevertheless, although the process parameters are adjusted, the reactor configuration is not always optimal. In fact, common phenomena that indicate non-ideal reactor behavior are frequently observed in industrial scale reactors, such as poor mixing, channeling or dead zones, which can result in unreacted substrate exiting the reactor. This might then have a severe impact on the downstream processing and on the respective operating costs of the process.

The complex reaction mechanisms of enzymatic processes and the difficulties in adapting the process to the reactor while simultaneously obtaining high reaction yields give a clear motivation to explore new strategies for improving a reactor configuration.

A new strategy for developing a reactor is therefore suggested in this paper: instead of adapting the process to a well-known reactor shape, a shape optimization method is used to find the reactor configuration with best performance for the specific reaction system.

Shape optimization is a mathematical methodology often used in mechanical and civil engineering. The methodology aims at finding the optimal geometry within a defined domain which minimizes or maximizes an objective function and fulfills specific pre-defined constraints. This work presents 
a new implementation of shape optimization in chemical engineering, which can become a novel approach for intensifying equipment and complements the existing process intensification methods. In the scientific literature, the majority of the shape optimization studies uses gradient-based optimization procedures [3-7]. However, there are some cases in which the optimization procedure consists of a gradient-free optimization method [8-10]. The gradient-based optimization method is an algorithm, which uses the direction of the steepest gradient of the objective function at the current point as the direction to evolve the subsequent iteration.

Gradient-free optimization methods are procedures which do not comprise derivatives in order to determine the optimum. These methods are often described as heuristic search methods, which mimic the process of natural selection.

The gradient-based optimization methods are described as efficient to determine the local minima for non-linearly constrained and convex problems. However, these methods cannot be used when solving discrete design variables or when using discontinuous functions and domains since for these cases it is not possible to calculate a gradient [11]. One solution to overcome such challenges is to use a gradient-free optimizer, or to start at different initial conditions in the parameter space. In this work, shape optimization is presented as the strategy to develop the configuration of an enzymatic reactor. The case study investigates the improvement of the reaction yield for a system using two parallel laminar inlet streams containing the enzyme and substrates respectively, by modifying the shape of a microreactor channel. The unfavorable thermodynamic conditions are included in the reaction mechanism. The residence time of the compounds inside the microreactor was maintained constant in order to normalize the results, and this normalization was practically achieved by modifying the inlet flow rate according to the volume changes. In this case, it is considered that the biocatalyst and one of the substrates are slowly diffusing compounds. The random search method was the shape optimization method chosen for improving the reactor 
configuration. Finding the optimized reactor shape computationally gives the opportunity to determine beforehand the optimal process conditions and optimal shape before performing experimental work, thus reducing the need for experimental work and the related consumption of raw materials.

\section{Methodology}

\subsection{Random search method description}

In the scientific literature several methods for optimization problems can be found. Random search is a simple gradient-free optimization method and it has been used and occasionally adopted for shape optimization in mechanical engineering $[12,13]$.

The random search algorithm will search randomly for an optimum over the domain of interest. Random search methods are widely applied to continuous as well as discrete optimization problems. Compared to deterministic methods, random search methods have the advantage of being applied to problems, where the objective function is non-convex (has more than one minimum), nondifferentiable, discontinuous, discrete or applied to a continuous-discrete domain [14]. Furthermore, the random search method is characterized as "robust" and it generates useful information in optimization problems relatively quickly.

The generic random search algorithm is defined by the following general optimization problem[15]:

Objective function:

$$
\Phi=\max _{\bar{x} \in S} f(\bar{x})
$$

Where $\bar{x}$ is the vector of $\mathrm{n}$ design variables, $S$ is an n-dimensional non-empty region and $f$ is the objective function. The objective function for random search may be a black-box function, i.e. the 
objective function does not need to be defined by a mathematical expression and can be a numerical value of $f$ which is returned for an input of $\bar{x}$.

The general random search algorithm is described by a sequence of points $\bar{x}_{i}$ on the iteration $i=0,1, \ldots k$ which may depend on the previous points and algorithm parameters. The vector $\bar{x}_{i}$ may represent a single point or a collection of points. The generic random search algorithm is given by: Step 0: Initialization of the random search with the initial vector $\bar{x}_{0} \subset S$ and iteration index $i=0$. Step 1: Generation of a collection of candidate points $\bar{y}_{i+1} \subset S$ according to a specific generator. Step 2: If $f\left(\bar{y}_{i+1}\right)<f\left(\bar{x}_{i}\right)$ a new local optimum set of points has been found. Update the vector $\bar{x}_{i}$ based on the optimum set of points $\bar{y}_{i+1}$.

Step 3: If a stopping criterion is met, stop the optimization routine. Otherwise increment $i$ and return to Step 1.

The generic random search algorithm is dependent on the procedure of generating the candidate points at Step 1 and on the update procedure in Step 2. A common method to generate a candidate point is to take a step size in a vector direction. Therefore, the Step 1 of the generic routine can be described by:

$$
\bar{y}_{i+1}=\bar{x}_{i}+k_{i} \bar{d}_{i}
$$

where the candidate point is taking a step from the current point $\bar{x}_{i}$ of length $k_{i}$ in a specified direction $\bar{d}_{i}$ on iteration $i$.

An option to generate the direction $\bar{d}_{i}$ that does not use any local information is to obtain the direction vector by sampling from a uniform distribution on an $n$-sphere. An $n$-sphere is the generalization of the ordinary sphere to spaces of arbitrary dimension [16]. The radius of an $n$ - 
sphere is defined by a set of points in a $(n+1)$-dimensional Euclidean space, which are at an equal distance $r$ (radius) from the central point. Hence, the n-sphere centered at the origin is defined by:

$$
S^{n}=\left\{x \in \mathbb{R}^{n+1}:\|x\|=r\right\}
$$

where $S^{n}$ is an $n$-dimensional manifold in an Euclidean $(n+1)$-dimensional space.

Specifically, a 0-sphere is the pair of points at the ends of a line segment, a 1-sphere corresponds to the circumference of the intersection of a disk and a plane and a 2-sphere corresponds to the two dimensional surface, which defines a three dimensional ball [16].

The step size can be generated randomly, and it may expand or shrink according to the success of the previous sample points.

\subsubsection{Shape optimization routine}

As described above, the shape optimization procedure was based on an evolutionary procedure, the random search method.

For an optimization, it is necessary to define the objective function and the constraints. In this study, the objective function is the concentration of product at the outlet of the microreactor, which must be maximized (alternatively the substrate concentration can be minimized). The optimization routine stops when either one of the constraints is satisfied or the system has converged. In this optimization problem, the routine will stop due to restrictions when the amount of product formed is ten times higher than the amount of product formed for the initial reactor configuration and the number of iterations between two local maxima does not exceed 1500. The number of iterations between two temporary optima was set to a maximum 1500 in order to limit the optimization time. The use of this constraint might stop the optimization cycle before finding the global optimum. 
However, it was necessary to compromise within the time and computational power available. It is possible that the random search method finishes when it finds a local optimum.

The formulation of this optimization problem is given in Equation 4:

$$
\begin{array}{ll}
\text { Maximize } & f\left(\bar{x}_{i}\right)=\int_{\Omega} r_{P}\left(\bar{x}_{i}\right) d V \\
\text { Subject to } & f\left(\bar{x}_{i}\right)=10 \times f\left(\bar{x}_{0}\right) \\
& f\left(\bar{x}_{i}\right) \leq f\left(\bar{x}_{i+e}\right) ; \quad e \leq 1500
\end{array}
$$

where $f$ is the objective function, $\bar{x}_{0}$ is the vector which sets the points for the initial geometry positions, $\bar{x}_{i}$ is the vector with the positions of the points, $i$ is the iteration number and $e$ is the number that evaluates the number of iterations between two local optima.

Using the notation defined previously the optimization procedure is defined by the following algorithm:

Until either the concentration of product is ten times higher or the number of iterations between two local optima is more than 1500 , run the following cycle:

-Initialize the random search with the initial vector $\bar{x}_{0}=\left\{x_{1}, x_{2}, \ldots x_{n}\right\} \subset S$, which gives the shape presented in Figure 1.

-Sample a new position for each of the elements of the vector $\bar{y}_{i+1}=\left\{y_{1}, y_{2} \ldots y_{n}\right\} \subset S$ from the 0 -sphere by the pair of points $\left\{x_{n}-0.03 x_{n}, x_{n}+0.03 x_{n}\right\}$ and by setting a random step size within the interval $[0,1]$.

- If $f\left(\bar{y}_{i+1}\right)>f\left(\bar{x}_{i}\right)$ a new local optimum set of points has been found. Set the vector $\bar{x}_{i}$ based on the optimum set of points $\bar{y}_{i+1}$.

-Interrupt the cycle when the concentration of product at the outlet is ten times higher or the number of iterations between local optima is more than 1500. Otherwise increment $i$ and return to the second step. 
The optimization routine consists of a finite volume analysis performed by ANSYS CFX ${ }^{\circledR}$ coupled with a MATLAB ${ }^{\circledR}$ routine, which includes the optimization procedure. ANSYS CFX ${ }^{\circledR}$ is a commercial computational fluid dynamics (CFD) software that is characterized as a "black box" software meaning that the user has no direct access to the mathematical equations of the fluid governing equations such as the Navier-Stokes equation, continuity equation and energy equation. Therefore, the user is not able to integrate the governing equations in the optimization routine. However, ANSYS CFX ${ }^{\circledR}$ differs from the other commercial CFD software packages by giving the opportunity to write the mesh files (software ICEM CFD ${ }^{\circledR}$ ), the simulation setup file (software CFX-Pre ${ }^{\circledR}$ ) and the post-processing files (software CFX-Post ${ }^{\circledR}$ ) as script files (See Figure 1). These script files are recorded files and are the basis for automatically establishing geometry, mesh, setup and post-processing procedures. The executing files for mesh, setup and solving are then generated from the script files.

The change of these script files, the generation of the executing files and the execution of the simulation can be performed via batch mode processes. With help of a user-programmed Matlab ${ }^{\circledR}$ code it is possible to establish commands for defining a run of a simulation in batch mode. It is furthermore possible to convert script files into arrays, modify system properties or points for definition of the geometry and command the start of a simulation. Therefore, there is a great opportunity to link and to establish a user-programmed and fully automated optimization routine between ANSYS CFX ${ }^{\circledR}$ and MATLAB ${ }^{\circledR}$.

It is important to emphasize that the two software programs have distinct roles: ANSYS CFX ${ }^{\circledR}$ solves the fluidic problem with the integrated reaction system and evaluates the product concentration at the outlet while MATLAB ${ }^{\circledR}$ executes the optimization method. The routine and interaction between ANSYS CFX ${ }^{\circledR}$ and MATLAB ${ }^{\circledR}$ used for the shape optimization routine is represented in Figure 1. 


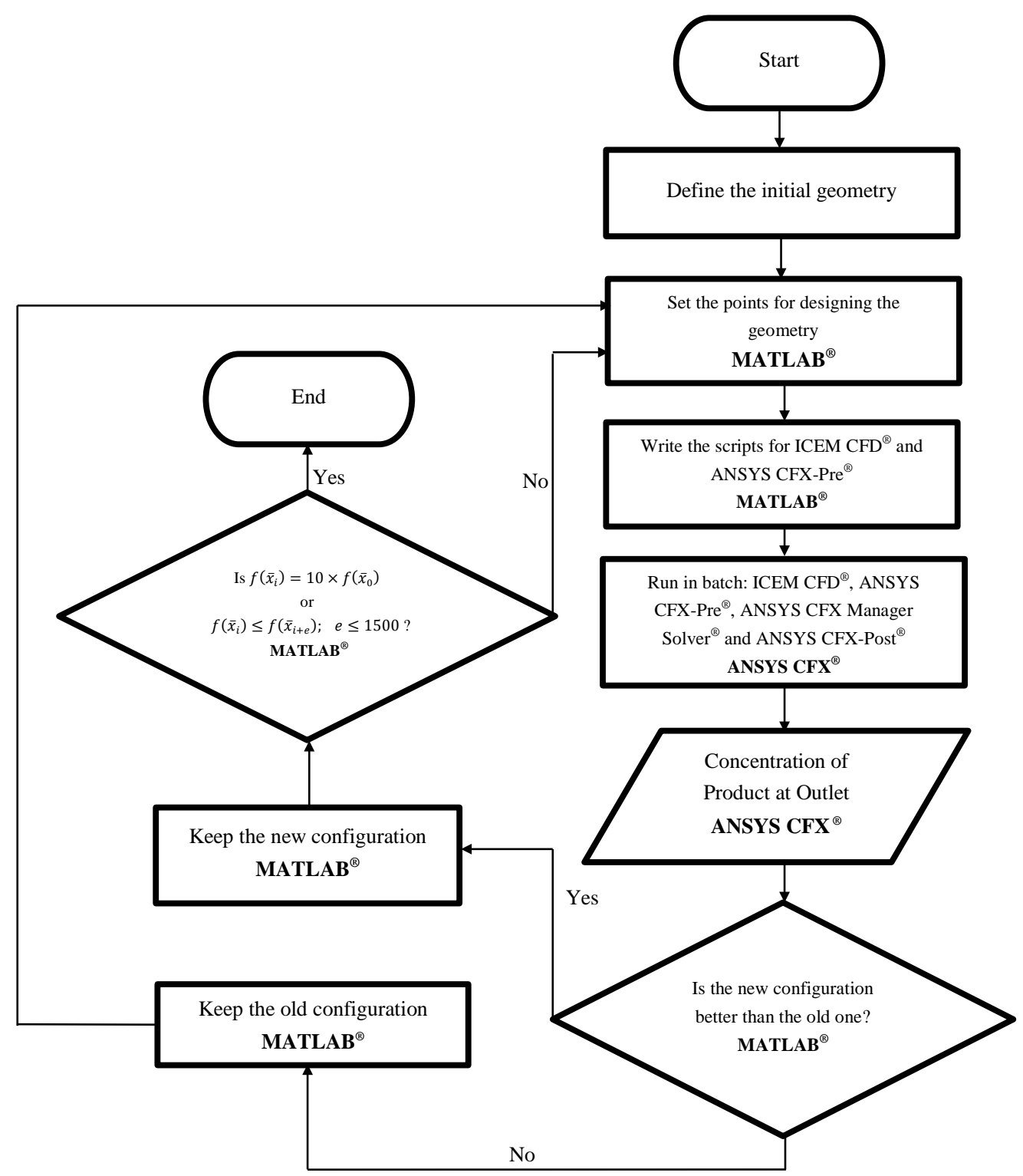

Figure 1 - Algorithm for the shape optimization of the enzymatic microreactor.

The optimization routine established in MATLAB ${ }^{\circledR}$ is characterized by five steps. The first step is to read information about the product concentration at the outlet of the reactor from the post processing file of ANSYS CFX ${ }^{\circledR}$. The second step is the decision on keeping the new geometry according to results read from the post processing file. The third step is the generation of a new set of points according to the random search procedure. Finally, the two last steps are the update of the reactor geometry and the flow rate in the CFD scripts and the start of the new CFD simulation. In shape optimization the alterations are directly made to the geometry and therefore the script file is 
updated for each iteration, which then creates the geometry and mesh (ICEM CFD ${ }^{\circledR}$ ). All the other set-up files are modified and generated automatically according to the changes of this new geometry/mesh file.

\subsection{Case study description}

The chosen case study investigates the optimization of the shape of the walls of a microreactor and evaluates how much the changes of the wall shape can improve the reaction yield. The microbioreactor is used for the production of optically pure chiral amines through an enzymatic reaction using amine transaminase (ATA). Chiral amines are important compounds in the synthesis of pharmaceutical drugs and agrochemicals $[17,18]$. Although chemical processes producing chiral amines already exist, the biocatalytic route has been considered as an important alternative process due to several advantages. The biocatalytic processes using enzymes are characterized by mild reaction conditions, high stereoselectivity and high enantioselectivity [19]. However, the reaction catalyzed by ATA is considered to be a very complex reaction with many challenges. One of the challenges with this biocatalytic reaction is the inhibition of amine transaminase by both the substrates and the products. Moreover, the ATA catalyzed reaction is also characterized by an unfavorable thermodynamic equilibrium, which drives the reaction towards the substrates [20]. The chosen model reaction is the synthesis of the chiral product (S)-1-phenylethylamine (PEA) and acetone (ACE) from acetophenone (APH) and isopropylamine (IPA) catalyzed by amine transaminase (ATA) as biocatalyst (Figure 2).

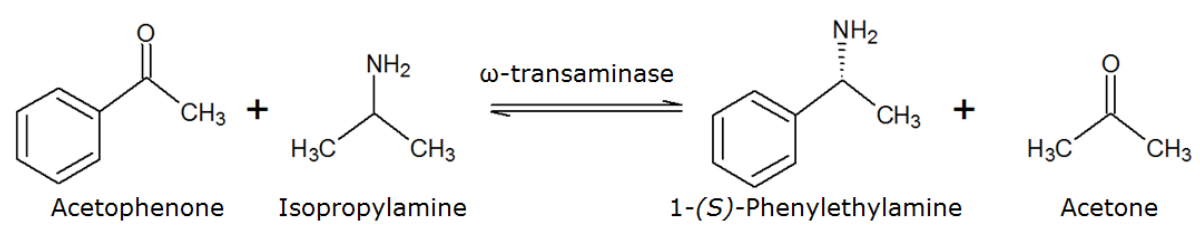

Figure 2 - Reaction system studied in the frame of microreactor optimization. 
ATA follows the Ping Pong Bi Bi kinetic mechanism in which one of the substrates (in this case, isopropylamine) binds first to the enzyme, and the co-product (acetone) is released before the second substrate (acetophenone) binds, and finally the product ((S)-1-phenylethylamine) is released from the enzyme site last. The kinetic rate equation for this reaction mechanism is given by [21]:

$$
\begin{aligned}
r_{P E A}=\frac{d[P E A]}{d t} & \frac{\left[E_{0}\right] k_{c a t}^{f} k_{c a t}^{r}\left([A P H][I P A]-\frac{[P E A][A C E]}{K_{e q}}\right)}{=} \frac{k_{c a t}^{f} K_{m}^{A P H}[I P A]\left(1+\frac{[P E A]}{K_{s i}^{P E A}}+\frac{[I P A]}{K_{S i}^{P I A}}\right)+k_{c a t}^{r} K_{m}^{I P A}[A P H]\left(1+\frac{[A P H]}{K_{S i}^{A P H}}+\frac{[A C E]}{K_{S i}^{A C E}}\right)+k_{c a t}^{f} \frac{K_{m}^{P E A}[A C E]}{K_{e q}}\left(1+\frac{[A P H]}{K_{S i}^{A P H}}+\frac{[A C E]}{K_{S i}^{A C E}}\right)}{k_{c a t}^{f} \frac{K_{m}^{A C E}[P E A]}{K_{e q}}\left(1+\frac{[P E A]}{K_{S i}^{P E A}}+\frac{[I P A]}{K_{S i}^{P P A}}\right)+k_{c a t}^{r}[A P H][I P A]+k_{c a t}^{f} \frac{K_{m}^{P E A}[I P A][A C E]}{K_{e q} K_{i}^{I P A}}+\frac{k_{c a t}^{f}[A C E][P E A]}{K_{e q}}+k_{c a t}^{f} \frac{K_{m}^{P P A}[A P H][P E A]}{K_{i}^{P E A}}}
\end{aligned}
$$

The specific kinetic parameters for this reaction system were determined and calibrated to the enzymatic mechanism by Al-Haque et al. (2012) [22]. Detailed information regarding the reaction mechanism and parameters can be found in Appendix A. In this case study, two compounds are considered as slowly diffusing compounds: ATA $\left(D=1 \cdot 10^{-11} \mathrm{~m}^{2} \mathrm{~s}^{-1}\right)$ and acetophenone $\left(\mathrm{D}=1 \cdot 10^{-12}\right.$ $\left.\mathrm{m}^{2} \mathrm{~s}^{-1}\right)$. ATA is considered to be a slow diffusing molecule due to its large molecular structure. Therefore, its diffusion coefficient was considered to be similar to the values reported for enzymes, $10^{-11} \mathrm{~m}^{2} \cdot \mathrm{s}^{-1}$ [23]. The slow diffusion of acetophenone in aqueous solution has been reported by Bodla et al. (2013) [24]. All the other compounds involved in this reaction system were considered to be fast diffusing substances and are assumed to have the same diffusion coefficient, $1 \cdot 10^{-9} \mathrm{~m}^{2} \cdot \mathrm{s}^{-1}$.

\subsubsection{Initial configuration of the microreactor}

The dimensions of the reactor in this case study are in the range of micrometers and therefore the flow is under microfluidic and laminar flow conditions. Microreactors are in general characterized by a very large surface-area-to-volume-ratio, very effective heat and mass transfer and enhanced 
control of process conditions due to automation. In microfluidics the flow is characterized by laminar flow, and therefore the mixing is essentially caused by diffusion. However, in this case study some of the compounds are characterized by low diffusion, and therefore the mass transfer within the reaction chamber is not very effective. This phenomenon gives the motivation to investigate the impact of shape optimization on the slow mixing velocity of acetophenone and ATA.

The dependency of the reaction in relation to the fluid mechanics of the system and the properties of the compounds will be evaluated by the governing equations of CFD i.e. the Navier-Stokes and the continuity equations represented below.

$$
\begin{gathered}
\text { Navier-Stokes equation: } \quad \frac{\partial}{\partial t} \rho \boldsymbol{v}=-(\boldsymbol{\nabla} \cdot \rho \boldsymbol{v} \boldsymbol{v})-\boldsymbol{\nabla} \cdot p-(\boldsymbol{\nabla} \cdot \boldsymbol{\tau}) \\
\text { Continuity equation: } \quad \frac{\partial \rho}{\partial t}=-\nabla(\rho \cdot \vec{v})+S
\end{gathered}
$$

The coupling with energy balances is not implemented since the reaction occurs under isothermal conditions.

The initial shape of the studied microreactor has the form of a YY-microchannel with a rectangular cross-section where the inlet and the outlet are located at the respective ends of the reactor. A YYmicrochannel is characterized by a long main reaction channel and by two channels at each extremity which meet at the beginning/end of the main channel making an angle lower than $90^{\circ}$, i.e. forming a Y shape (See Figure 3).

The Y shapes at each end of the reaction chamber correspond to the inlet and the outlet where two streams meet at the entrance of the main channel and are again split into two streams at the exit of the channel. In one inlet of the microreactor, a solution with ATA is introduced, and a solution of 
acetophenone and isopropylamine enters in the other inlet (See Figure 3). The concentrations of the reactants and enzyme can be found in Appendix A.

The optimization routine focuses on the modification of the surface of the main channel of a microreactor. The main channel consists of a chamber without divisions. The initial configuration has the following dimensions: $0.25 \mathrm{~mm}$ of width for each inlet and outlet, $1 \mathrm{~mm}$ of height and 100 mm of length (see Figure 3).

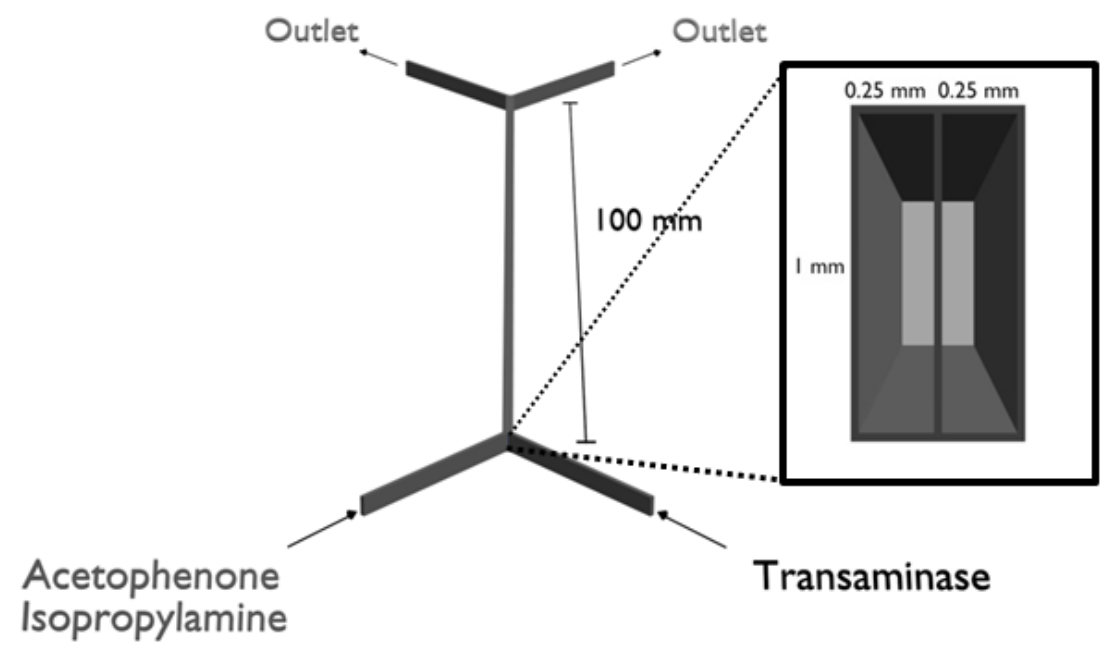

Figure 3 - Microreactor configuration with indication of the inlets and outlets and the substances introduced at each inlet with a detailed view of the inlet of the reaction channel.

The shape optimization routine makes changes on small vertical sections of the reaction chamber measuring $1 \mathrm{~mm}$ in length. These vertical sections are characterized by a closed spline, which connects a group of 14 points. These splines are linked to form the surface of the channel (see Figure 4a)).

The distance between the closed splines is very small, and if the whole channel is considered, the optimization problem would be excessively large in terms of computational effort. This study therefore focused only the first section of the reaction channel $(10 \mathrm{~mm})$. 
The points on the surface of the considered section (140 points) are modified according to the random search procedure. The closed splines are modified due to the alteration of the disposition of the points and consequently the shape of the channel is also modified. The points which are on the lateral parts of the channel can be moved horizontally, the points at the top and bottom of the channel can be moved vertically and the corner points can be moved in both vertical and horizontal directions (See Figure 4b)). The residence time inside the section of the microreactor is 3 minutes for all simulations. Since the volume of the microreactor varies from simulation to simulation, the flowrate has to be adjusted in order to maintain the residence time constant.

a)

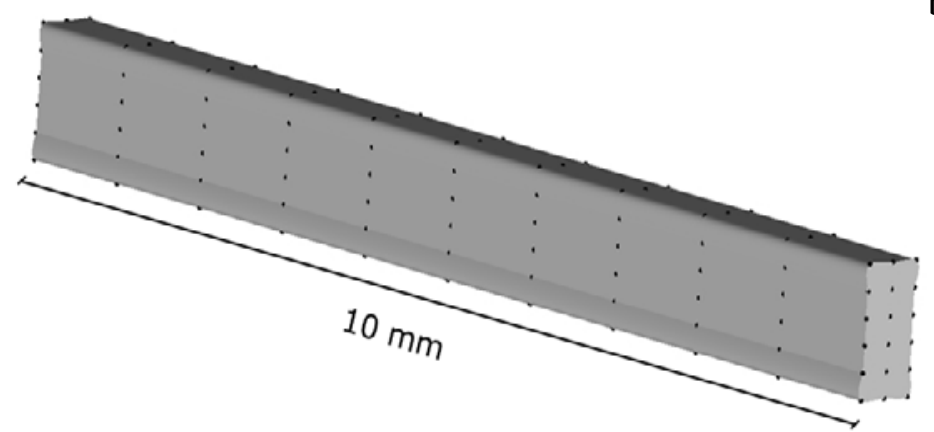

b)

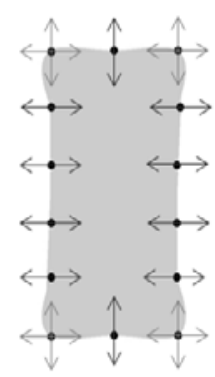

Figure 4 - a) YY-microreactor configuration; b) Structure to be optimized with all surface points represented and the directions for modification of the points indicated by arrows.

The microreactor geometry has been discretized in small volume elements creating a mesh. The generation of the mesh and discretization of the domain into elements allows the numerical solution of the governing equations of the CFD code which provides discrete values of the equations for each mesh node. The mesh is one important part of the CFD simulation since the quality of the mesh matters for the solution of the CFD analysis. Therefore, the mesh generation is usually a complicated and time consuming process.

The mesh generation method has been recorded in a script file when the mesh was generated for the initial shape. The method for generating the mesh is the same for all the shapes given by the random 
search method. The mesh adapts itself to all the different geometries and it is ensured that the mesh covers the whole microreactor structure. In this way it is possible to achieve consistency and automation of the mesh generation without user interference.

The used mesh is a structured mesh for which the geometry is discretized in quadrilateral elements in 2D and hexahedral elements in 3D. All microreactor configurations were discretized in 97944 hexahedral elements. A structured mesh presents several advantages compared to an unstructured one (tetrahedral elements) such as higher accuracy of the simulation - especially for flows with high gradients, better convergence and fewer mesh elements and therefore lower computation time and memory usage [25]. By adopting this type of mesh, the domain became discontinuous, which means not all combinations of points will be possible.

The discontinuity of the domain is caused by the formation of a sharp angled geometry for some combinations of points and therefore the elements in that zone tend to be tetrahedral and stop being hexahedral. The quality of the mesh is evaluated by the angles of the elements, and should be sufficient to fulfill the solver requirements.

a)

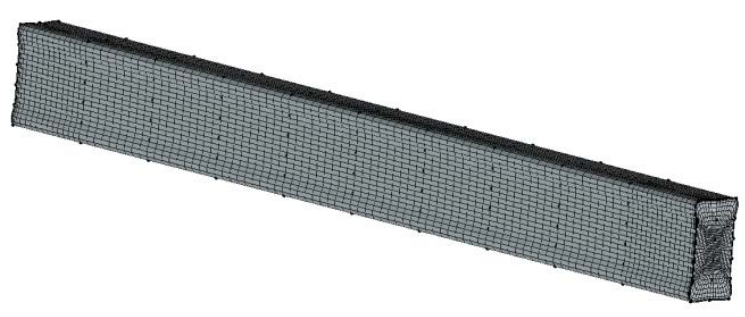

b)

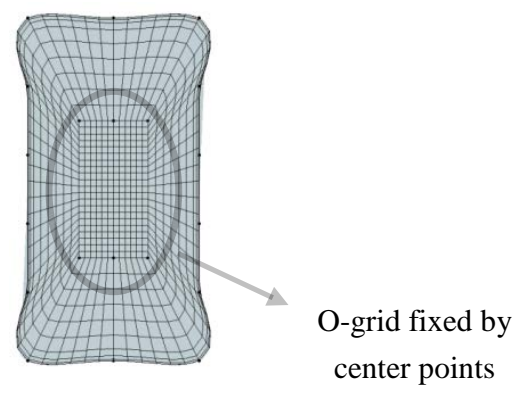

Figure 5 - a) applied mesh strategy for a microreactor with shape optimization for the initial shape. b) Ogrid application to the mesh of the microreactor 
The ANSYS CFX ${ }^{\circledR}$ compatible mesh generator, ICEM CFD ${ }^{\circledR}$ has the option to verify the mesh quality. The approach used to evaluate the quality was the named Determinant method. This method computes the deformation of the elements in the mesh by calculating the Jacobian matrix of each hexahedron and then normalizes the determinant of the matrix. A value of 1 represents a perfect hexahedral cube, while a value equal to 0 corresponds to an inverted cube with a negative volume. In general, determinant values above 0.3 are acceptable for the solver method used here. The created geometry does not have a rectangular cross-section. In fact the corners of the cross-section are curved.

A curvilinear mesh called O-grid in ICEM CFD ${ }^{\circledR}$, which is usually applied to curved shapes, was applied to the faces of the inlet and outlet as shown in Figure 5a) in order to minimize the discontinuity of the domain. The center points of the O-grid, which correspond to the corners of the perfect hexahedral elements of the structure, have been fixed in position for the initial shape of the microreactor. In this way it was possible to avoid sharp angles inside the structure (see Figure 5b)). Despite all the efforts to avoid discontinuous domains, there were still several shapes for which it was not possible to solve the system due to poor mesh quality. Therefore, the shapes with bad quality mesh were not considered in this study.

\section{Results and Discussion}

This optimization study included in total 9296 simulations, and each simulation took between 2-5 minutes to solve. The best achieved shape has the aspect of a wavy structure on all four walls of the channel. In the initial configuration, $4.4 \cdot 10^{-5} \mathrm{mM}$ of substrate were converted. 


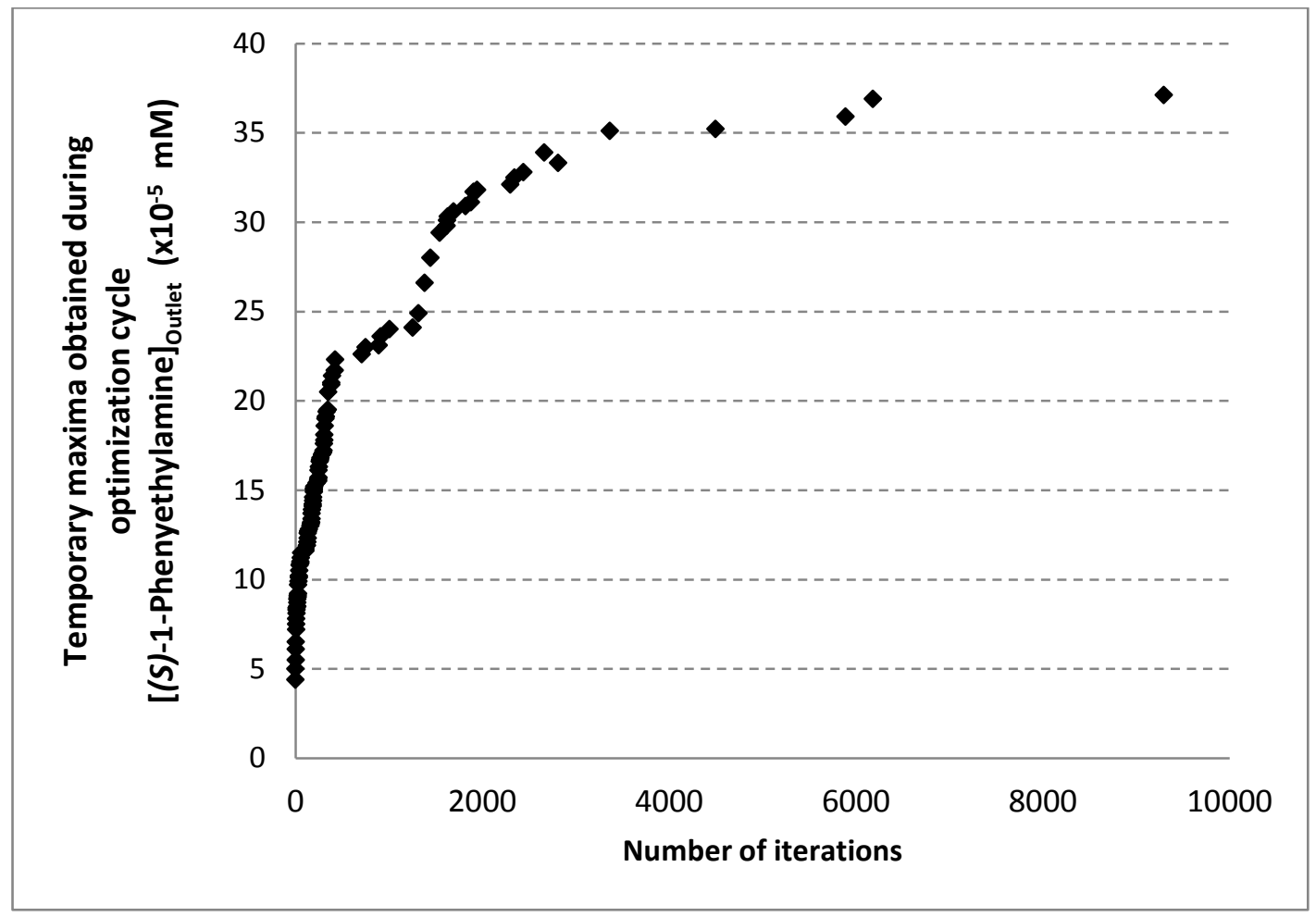

Figure 6 - Concentration of phenylethylamine at the outlet of the microreactor for all the temporary optimal configurations obtained during the optimization cycle.

The low conversion inside the channel was expected due to the slow diffusion of the enzyme and acetophenone which results in a very thin interface between the parallel streams where the reaction occurs. Figure 6 shows the phenylethylamine concentration at the outlet of all the temporary optimal configurations obtained plotted along the iterations of the optimization cycle. One should note that in the beginning of the optimization it was easy to find a new optimal configuration, however at the end of the cycle the number of iterations required to obtain a better configuration increased exponentially. The changes made to the surface of the reactor channel resulted in sharp angled geometries which have a negative impact on the mesh and prevented the simulations from running as explained before. In the end of the cycle the microreactor shape was very complex and more likely to form sharp angles. Thus, the shape was restricted to fewer geometries which could be simulated and it became more difficult to find the next optimum. The optimization routine ended up stopping because of the condition of limiting the number of iterations between two local optima to 
1500. In the final configuration, the reaction yield was 8.4 times the yield of the initial shape corresponding to $37.1 \cdot 10^{-5} \mathrm{mM}$ of product formed (see Figure 6 ). The achieved improvement was very close to the pre-defined goal, which was to achieve 10 times the yield of the initial shape.

A previous study using topology optimization on a microreactor [26] has shown an improvement of 10 fold which is similar to the result obtained in this study. These results show that the optimization of the shape and the topology of microreactors is high impact strategies for designing microreactors.

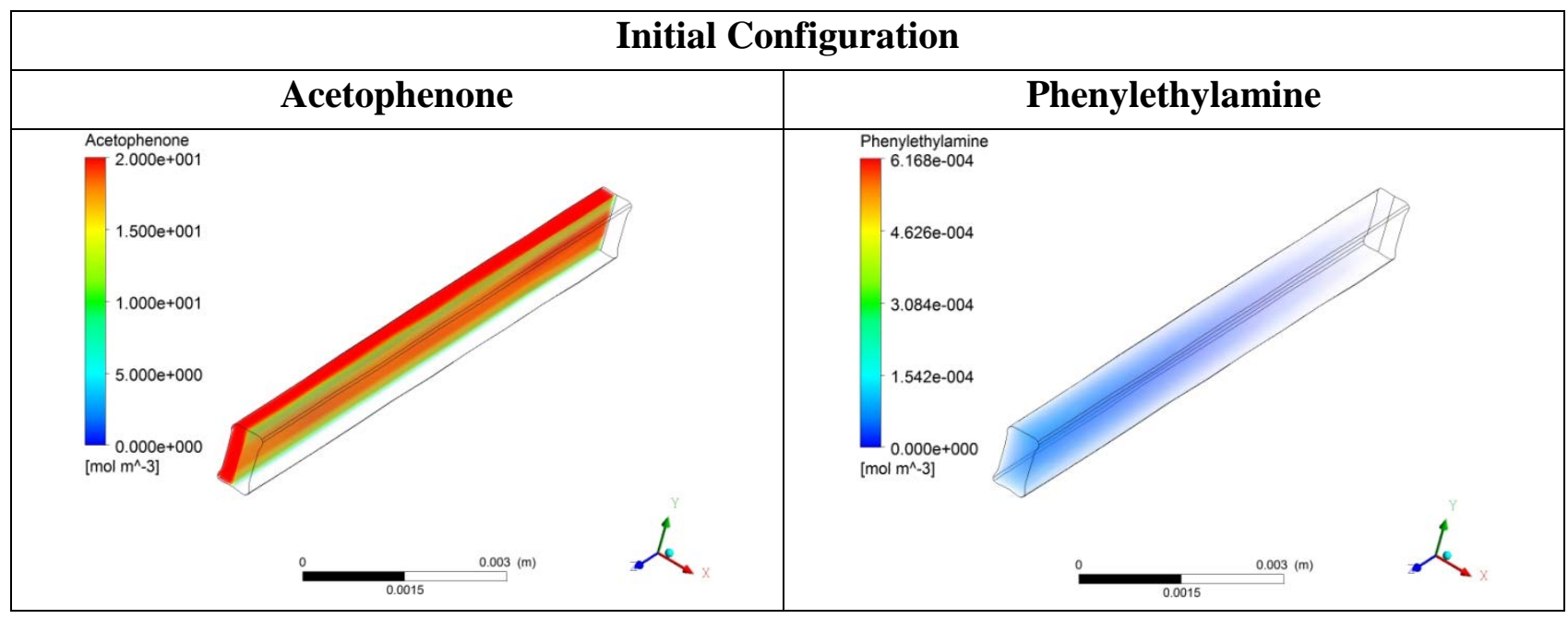

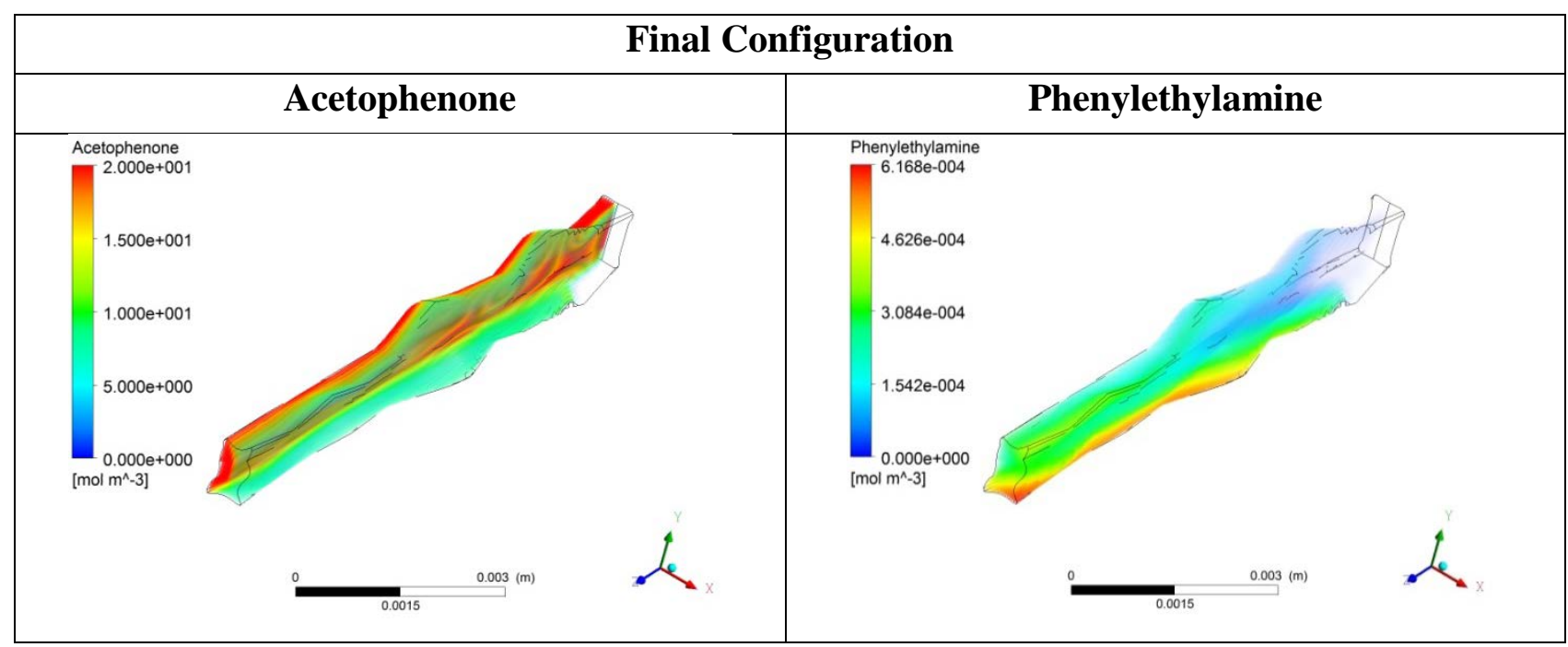

Figure 7 - Concentration of acetophenone and phenylethylamine along the channel for initial and final configurations. 
The comparison of the results between the initial configuration and the final configuration for the substrate consumption and the product formation can be found in Figure 7. The comparison between the geometry of the initial and the final microreactor configurations can be seen in Figure 8. The final configuration is characterized by a series of expansions and shrinkages of the surface of the microreactor. From Figure 7 and Figure 8, it is possible to verify that the curvatures resulting from the optimization procedure have a significant influence on the mixing of the parallel streams and consequently on the reaction yield.

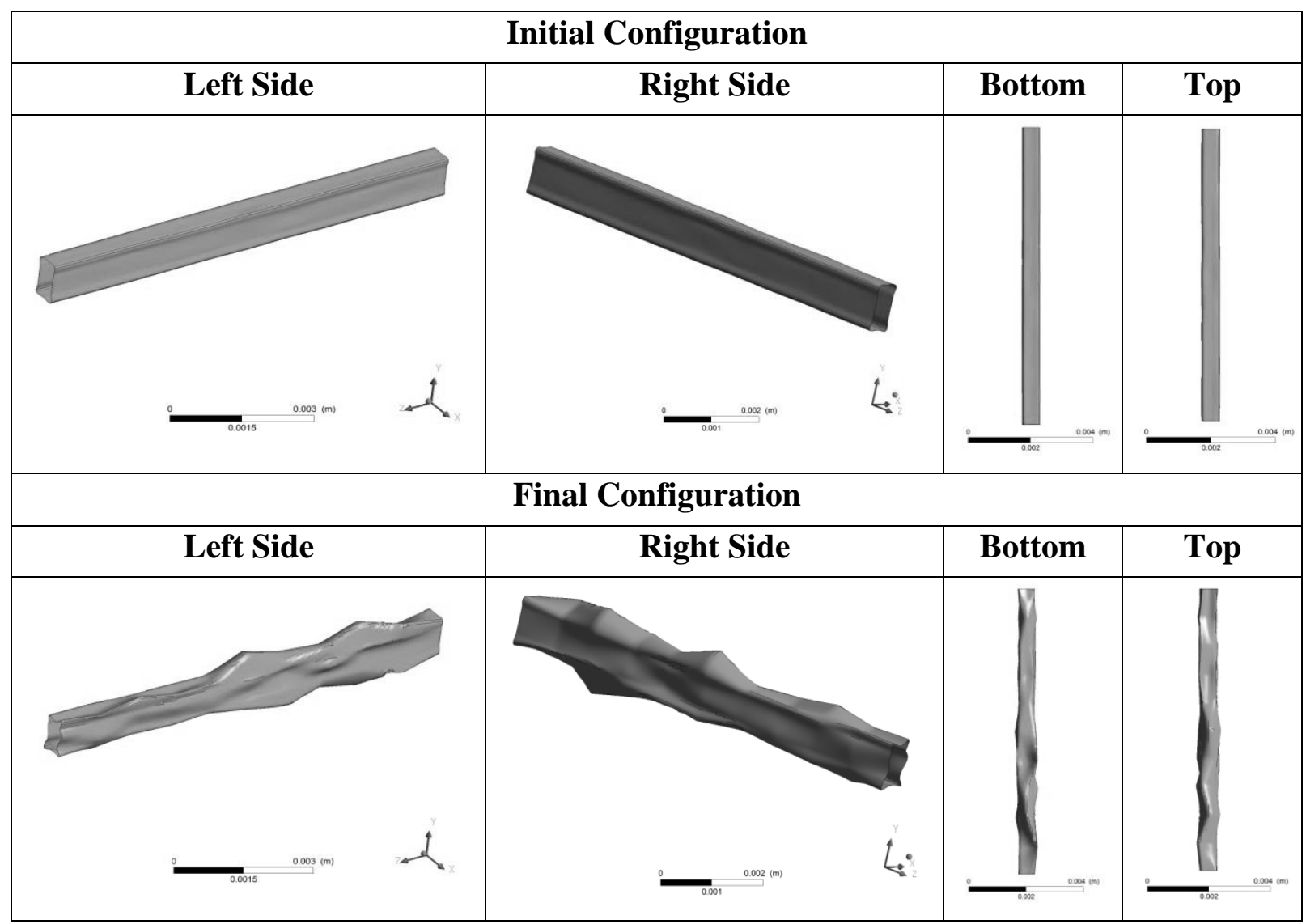

Figure 8 - Views from the four directions of the initial and final configurations

It is also possible to verify that the bottom and the top surfaces changed more than the lateral surfaces. The displacement of the points on the top and the bottom seems to have a higher importance than the movement of the points on the lateral surfaces. 
From the bottom view, it is possible to see that a deep and narrow expansion contributes greatly to the mixing of the two streams and consequently to the product formation. The narrowing of the channel contributes to shorter diffusion distances of the compounds, and therefore to the mixing and consequently, to a higher reaction yield. From the top view, the channel contributes to the mixing inside by introducing a passive mixing form similar to a staggered herringbone structure. The staggered herringbone and similar structures have been widely applied in micromixers and reported in the scientific literature $[27,28]$. This kind of structures introduces helix shaped streamlines which have a considerable impact on the mixing of parallel streams [29]. The analysis of the results suggests that in this case the mixing plays an important role for achieving higher yields. The resulting structure of shape optimization of the YY-microreactor is very complex. The complexity of the shape makes the fabrication of the structure very difficult or impossible and therefore it is not feasible to investigate the final microreactor design experimentally. The achievement of this complex structure was caused by the high number of design variables (points distributed on the microreactor surface), by the various combinations for displacement of the points and by the discontinuous domain, which withholds the solution of geometries that did not fulfill the mesh solver requirements. However, this study allows gathering information which can be useful for the future fabrication of improved microreactors with integrated mixing.

\section{Conclusions}

This article presents an innovative application of shape optimization which was used for optimizing a three-dimensional geometry of a reactor. This novel reactor intensification procedure allowed the evaluation of the influence of the modification of a microreactor channel shape on the reaction yield. 
The optimization routine is a link between two software packages: ANSYS CFD ${ }^{\circledR}$ and MATLAB ${ }^{\circledR}$. The user can record ANSYS CFD ${ }^{\circledR}$ script files which establish the geometry, mesh, simulation setup, simulation solution and post processing. On the other hand MATLAB ${ }^{\circledR}$ makes the changes to the script files according to the random search method which is also implemented by MATLAB ${ }^{\circledR}$. The final configuration cannot be easily fabricated due to the complexity of the resulting structure. However, the shape optimization of the microreactor allows the collection of information on how to build a microreactor with a shape that promotes the reaction. Upon the start of the implementation of the case study, there was no information on the impact of the complex kinetics on the reaction yield. From the results of shape optimization, it was possible to identify that the mixing by convection is the most important phenomenon for the product formation in this specific case. The results of this case study are in fact a validation of this shape optimization method since the final flow configuration resembles the flow in a staggered herringbone structure, which is a very wellknown well-performing passive mixer.

The final conclusion from this study is that shape optimization allows the investigation of potential intensification strategies in order to create improved reactor designs. Although in this case it is not possible to fabricate the final configuration due to the high number of design variables (surface points), nonetheless, this challenge can be overcome by simplifying the shape optimization method. This simplification can be achieved by implementing more restrictions in the optimization procedure which consider the feasibility of fabrication.

\section{Acknowledgements}

The research leading to these results has received funding from the European Union FP7 (FP7/2007-2013) Project BIOINTENSE - Mastering Bioprocess integration and intensification 
across scales (Grant Agreement number 312148) and from Novo Nordisk Fonden (project 'Exploring biochemical process performance limits through topology optimization').

\section{Bibliography}

[1] J.M. Woodley, M. Breuer, D. Mink, A future perspective on the role of industrial biotechnology for chemicals production, Chem. Eng. Res. Des. 91 (2013) 2029-2036.

[2] H.S. Fogler, Elements of chemical reaction engineering, fourth ed., Prentice Hall PTR, New Jersey, 2005.

[3] K.C. Giannakoglou, K.C. Papadimitrou, Adjoint Methods for Shape Optimization, D. Trévenin, G. Janiga (Eds.), Optimization and Computational Fluid Dynamics, first ed., Springer-Verlag, Berlin, 2008: pp. 79-108.

[4] G. Allaire, A.-M. Toader, F. Jouve, A Level-Set Method for Shape Optimization, C. R. Math. 334 (2009) 1125-1130.

[5] C.A. Mader, J.R.R.A. Martins, Computing Stability Derivatives and Their Gradients for Aerodynamic Shape Optimization, AIAA J. 52 (2014) 2533-2546.

[6] S. Hossain, A. Husain, K.-Y. Kim, Shape optimization of a micromixer with staggeredherringbone grooves patterned on opposite walls, Chem. Eng. J. 162 (2010) 730-737.

[7] A. Afzal, K.-Y. Kim, Optimization of pulsatile flow and geometry of a convergentdivergent micromixer, Chem. Eng. J. 281 (2015) 134-143.

[8] E. Kita, 2D shape optimization using genetic algorithm, Comput. Assist. Mech. Eng. Sci. 5 (1998) 311-321.

[9] T.L. Holst, T.H. Pulliam, Aerodynamic Shape Optimization Using A Real-NumberEncoded Genetic Algorithm, 19th Appl. Aerodyn. Conf. (2001) 1-14. 
[10] A. Oyama, Aerodynamic Optimization of Transonic Wing Design Based on Evolutionary Algorithm, Third Int. Conf. Nonlinear Probl. Aviat. Aerosp. Proc., 2002, pp. 537-546.

[11] D.W. Zingg, M. Nemec, T.H. Pulliam, A comparative evaluation of genetic and gradientbased algorithms applied to aerodynamic optimization, Rev. Eur. Mécanique Numérique. 17 (2008) 103-126.

[12] J. Haslinger, D. Jedelský, T. Kozuker, J. Tvrdík, Genetic and random search methods in optimal shape design problems, J. Glob. Optim. 16 (2000) 109-131.

[13] S.A. Rukolaine, Shape optimization of radiant enclosures with specular-diffuse surfaces by means of a random search and gradient minimization, J. Quant. Spectrosc. Radiat. Transf. 151 (2015) 174-191.

[14] Z.B. Zabinsky, Stochastic adaptive search for Global optimization, first ed., Kluwer Academic Publishers, New York, 2003.

[15] Z.B. Zabinsky, Random Search Algorithms, J. J. Cochran (Ed.), Wiley Encyclopedia of Operations Research and Management Science, second ed., John Wiley and Sons, Chichester, 2010.

[16] G. Marsaglia, Choosing a Point from the Surface of a Sphere, Ann. Math. Stat. 43 (1972) 645-646.

[17] D. Ghislieri, N.J. Turner, Biocatalytic Approaches to the Synthesis of Enantiomerically Pure Chiral Amines, Top. Catal. 57 (2014) 284-300.

[18] M. Höhne, U.T. Bornscheuer, Biocatalytic Routes to Optically Active Amines, ChemCatChem. 1 (2009) 42-51. 
[19] D. Koszelewski, I. Lavandera, D. Clay, D. Rozzell, W. Kroutil, Asymmetric Synthesis of Optically Pure Pharmacologically Relevant Amines Employing $\omega$-Transaminases, Adv. Synth. Catal. 350 (2008) 2761-2766.

[20] P. Tufvesson, J. Lima-Ramos, J.S. Jensen, N. Al-Haque, W. Neto, J.M. Woodley, Process considerations for the asymmetric synthesis of chiral amines using transaminases, Biotechnol. Bioeng. 108 (2011) 1479-1493.

[21] V. Leskovac, Comprehensive enzyme kinetics, first ed., Kluwer Academic Publishers, New York, 2003.

[22] N. Al-Haque, P.A. Santacoloma, W. Neto, P. Tufvesson, R. Gani, J.M. Woodley, A robust methodology for kinetic model parameter estimation for biocatalytic reactions, Biotechnol. Prog. 28 (2012) 1186-96.

[23] L.T. Kremzner, I.B. Wilson, A partial characterization of acetylcholinesterase, Biochemistry. 3 (1964) 1902-1905.

[24] V.K. Bodla, R. Seerup, U. Krühne, J.M. Woodley, K. V. Gernaey, Microreactors and CFD as Tools for Biocatalysis Reactor Design: A case study, Chem. Eng. Technol. 36 (2013) 1017-1026.

[25] J.F. Thompson, B.K. Soni, N.P. Weatherill, Handbook of grid generation, first ed., CRC Press, Roca Baton, 1999.

[26] D. Schäpper, R. Lencastre Fernandes, A. Eliasson Lantz, F. Okkels, H. Bruus, K.V. Gernaey, Topology optimized microbioreactors, Biotechnol. Bioeng. 108 (2011) 786-796 [27] V. Hessel, H. Löwe, A. Müller, G. Kolb, Chemical micro process engineering: processing and plants, first ed., Wiley-VCH, Weinheim, 2005. 
[28] D.G. Hassell, W.B. Zimmerman, Investigation of the convective motion through a staggered herringbone micromixer at low Reynolds number flow, Chem. Eng. Sci. 61 (2006) 2977-2985.

[29] A.D. Stroock, S.K.W. Dertinger, A. Ajdari, I. Mezic, H.A. Stone, G.M. Whitesides, A.D. Stroock, Chaotic mixer for microchannels, Science. 295 (2002) 647-651.

\section{Appendix A}

The reaction mechanism is characterized by 14 parameters, which include the reaction rate constants, the Michaelis-Menten constants, the product and substrate inhibition constants and the equilibrium constant of the reaction. The rate equation describes both the forward reaction towards product formation (phenylethylamine and acetone) and the reverse reaction towards the substrate formation (acetophenone and isopropylamine). The Equation 4 represents the reaction mechanism of the amino transaminase and is characterized by the Ping Pong Bi Bi mechanism. This equation represents both the forward reaction towards the products, (S)-1-phenylethylamine (PEA) and acetone (ACE), and the reverse reaction towards the substrates, acetophenone (APH) and isopropylamine (IPA). In the reaction rate equation, the turnover number of the forward reaction is represented by $k_{c a t}^{f}$ and the turnover number of the reverse reaction is denoted by $k_{c a t}^{r}$. The rate equation includes also the Michaelis-Menten constant for each of the compounds participating in the reaction. The Michaelis-Menten constants for acetophenone, isopropylamine, $(S)$-1phenylethylamine and acetone are denoted by $K_{M}^{A P H}, K_{M}^{I P A}, K_{M}^{P E A}$ and $K_{M}^{A C E}$, respectively. This specific reaction is greatly influenced by competitive substrate inhibition of acetophenone and (S)-1-phenylethylamine. The substrate inhibition constants for both acetophenone and (S)-1phenylethylamine are represented by $K_{S i}^{A P H}$ and $K_{S i}^{P E A}$, respectively. The significance of substrate 
inhibition by acetone $\left(K_{S i}^{A C E}\right)$ and isopropylamine $\left(K_{S i}^{I P A}\right)$ was found to be negligible. The core inhibition constants for acetophenone, isopropylamine, (S)-1-phenylethylamine and acetone are represented in the kinetic equation by $\left(K_{S i}^{A P H}\right),\left(K_{S i}^{I P A}\right),\left(K_{S i}^{P E A}\right)$ and $\left(K_{S i}^{A C E}\right)$. The equilibrium constant is given by $K_{e q}$. The parameters $\gamma$ and $\lambda$ are the binary reaction direction indicators. The parameters, estimated by Al-Haque et al. [22] and used in these simulations, are summarized in Table A1.

Table A1 - Parameters of the kinetic model estimated by Al-Haque et al. [22].

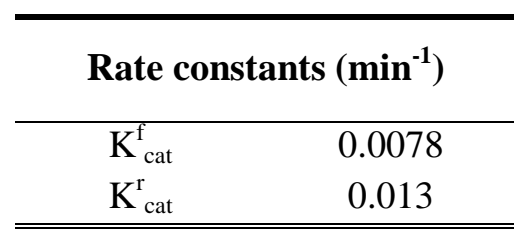

Michaelis-Menten constants

\begin{tabular}{cc}
\multicolumn{2}{c}{$(\mathbf{m M})$} \\
\hline $\mathrm{K}_{\mathrm{M}}{ }^{\mathrm{APH}}$ & 1.85 \\
$\mathrm{~K}_{\mathrm{M}}{ }^{\mathrm{IPA}}$ & 101.28 \\
$\mathrm{~K}_{\mathrm{M}}{ }^{\mathrm{ACE}}$ & 148.99 \\
$\mathrm{~K}_{\mathrm{M}}{ }^{\text {PEA }}$ & 0.12 \\
\hline \hline \multicolumn{2}{c}{ Equilibrium constant } \\
\hline $\mathrm{K}_{\mathrm{EQ}}$ & 0.033
\end{tabular}

\begin{tabular}{cc}
\hline \multicolumn{2}{c}{$\begin{array}{c}\text { Substrate inhibition } \\
\text { constants } \\
(\mathbf{m M})\end{array}$} \\
\hline $\mathrm{K}_{\mathrm{Si}}^{\mathrm{APH}}$ & 4.15 \\
$\mathrm{~K}_{\mathrm{Si}}^{\mathrm{PEA}}$ & 10.38 \\
\hline \multicolumn{2}{c}{ Core inhibition constants } \\
\multicolumn{2}{c}{ (mM) } \\
\hline $\mathrm{K}_{\mathrm{i}}^{\mathrm{APH}}$ & 0.09 \\
$\mathrm{~K}_{\mathrm{i}}^{\mathrm{IPA}}$ & 4281 \\
$\mathrm{~K}_{\mathrm{i}}^{\mathrm{ACE}}$ & 0.11 \\
$\mathrm{~K}_{\mathrm{i}}^{\mathrm{PEA}}$ & 105
\end{tabular}

The concentrations of the substrates and the enzyme at the inlet before mixing the flows are presented in Table A2:

Table A2 - Concentration of substrates and enzyme at the inlet of the microreactor.

\begin{tabular}{cc}
\hline Substance & Concentration \\
\hline Acetophenone & $20 \mathrm{mM}$ \\
Isopropylamine & $1 \mathrm{M}$ \\
$\omega$-transaminase & $0.15 \mathrm{M}$ \\
\hline
\end{tabular}

The choice of the substrate and enzyme concentrations considered in this study was made based on experimental work of Bodla et al. [24]. The concentration of enzyme was calculated from previously cited information available in the scientific literature $(6 \mathrm{mg} / \mathrm{mL})$ and assuming that the 
molecular weight of the enzyme is $40 \mathrm{kDa}$ and the mass of enzyme preparation contained exclusively pure enzyme. 